\title{
JPEG XL next-generation image compression architecture and coding tools
}

Jyrki Alakuijala, Ruud van Asseldonk, Sami Boukortt, Martin Bruse, Iulia-Maria Comșa, et al.

Jyrki Alakuijala, Ruud van Asseldonk, Sami Boukortt, Martin Bruse, lulia-Maria Comșa, Moritz Firsching, Thomas Fischbacher, Evgenii Kliuchnikov,

Sebastian Gomez, Robert Obryk, Krzysztof Potempa, Alexander Rhatushnyak, Jon Sneyers, Zoltan Szabadka, Lode Vandevenne, Luca Versari, Jan Wassenberg, "JPEG XL next-generation image compression architecture and coding tools," Proc. SPIE 11137, Applications of Digital Image Processing XLII, 111370K (6 September 2019); doi:

$10.1117 / 12.2529237$

Event: SPIE Optical Engineering + Applications, 2019, San Diego, California, United States 


\title{
JPEG XL next-generation image compression architecture and coding tools
}

\author{
Jyrki Alakuijala ${ }^{a}$, Ruud van Asseldonk ${ }^{\dagger, a}$, Sami Boukortt ${ }^{\mathrm{a}}$, Martin Bruse ${ }^{\mathrm{a}}$, Iulia-Maria \\ Comșa $^{a}$, Moritz Firsching ${ }^{a}$, Thomas Fischbacher ${ }^{a}$, Evgenii Kliuchnikova ${ }^{a}$, Sebastian Gomez ${ }^{a}$, \\ Robert Obryk ${ }^{\mathrm{a}}$, Krzysztof Potempa ${ }^{\mathrm{a}}$, Alexander Rhatushnyak ${ }^{\dagger, \mathrm{a}}$, Jon Sneyers ${ }^{\mathrm{b}}$, Zoltan \\ Szabadka $^{\dagger, a}$, Lode Vandevenne ${ }^{\text {, }}$, Luca Versari ${ }^{*}$, , and Jan Wassenberg ${ }^{\text {a }}$

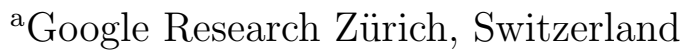 \\ ${ }^{\mathrm{b}}$ Cloudinary, Israel
}

\begin{abstract}
An update on the JPEG XL standardization effort: JPEG XL is a practical approach focused on scalable web distribution and efficient compression of high-quality images. It will provide various benefits compared to existing image formats: significantly smaller size at equivalent subjective quality; fast, parallelizable decoding and encoding configurations; features such as progressive, lossless, animation, and reversible transcoding of existing JPEG; support for high-quality applications including wide gamut, higher resolution/bit depth/dynamic range, and visually lossless coding. Additionally, a royalty-free baseline is an important goal. The JPEG XL architecture is traditional block-transform coding with upgrades to each component. We describe these components and analyze decoded image quality.
\end{abstract}

Keywords: JPEG XL, image compression, DCT, progressive

\section{INTRODUCTION}

JPEG $^{1}$ has reigned over the field of practical lossy image compression ever since its introduction 27 years ago. Other mainstream media encodings, especially for video and sound, have gone through several generations of significant and well-rounded improvements during the same time period. One can ask what are the characteristics of JPEG and the field of lossy image compression that let JPEG keep its position in spite of all the efforts so far. We have identified two main repeating issues in previous efforts attempting to replace JPEG with more efficient image encodings, namely, psychovisual performance in professional quality photography and the lack of gradual migration features. We have addressed them thoroughly in our new proposal, JPEG XL, as well as generally improved compression performance.

As an outcome of the 79th JPEG meeting in April 2018, the JPEG Committee announced the JPEG XL activity, ${ }^{2}$ aiming to standardize a new generation of image coding that offers substantially better compression efficiency than existing image formats (e.g. 50\% size reduction over JPEG), along with features desirable for web distribution and efficient compression of high-quality images. Proposals were due September 1, 2018, and based on psychovisual experiments, Google's PIK $^{3}$ and Cloudinary's FUIF ${ }^{4}$ codecs were chosen as the base framework for JPEG XL and its further core experiments. As of July 2019, JPEG XL has advanced into the committee draft phase, and is being prepared for circulation in ISO's national bodies.

The authors believe that a key factor in the failure to deliver outstanding high quality psychovisual performance in new lossy image codecs has been their tendency to target their best performance at too low bit rates, and the coding methods not extending to higher bit rates without losing most of their efficiency along the way. This can be most easily observed in image codecs that are derived from successful video encoding research. To make sure that we get this right with JPEG XL, we have gathered real-world usage data on existing JPEG utilization, and conducted independent experiments on how this could be mapped into bit rates and quality settings in JPEG XL. We note that as a result of this focus we have aimed at higher image qualities and bit rates than previous efforts.

\footnotetext{
$\dagger$ Work done while the author was at Google.

* veluca@google.com
}

Applications of Digital Image Processing XLII, edited by Andrew G. Tescher, Touradj Ebrahimi, Proc. of SPIE Vol. 11137, 111370K (C) The Authors. Published under a Creative Commons Attribution CC-BY 3.0 License · doi: 10.1117/12.2529237 
Migrating from the previous generation of media codecs to the next generation introduces a technology transition cost. Two or more incompatible encodings of the same original data need to be maintained and managed for different clients, often increasing storage capacity cost. Occasionally some fragmentation happens already within a single generation of codecs, contributing additionally to the transition cost. On-line transcoding, while practical in avoiding storage costs, has disadvantages. It introduces computational cost and additional quality and efficiency losses. These transcoding losses can be unpredictable and more dramatic if the original in previous generation technology has been compressed lossily to the highest possible degree, because compression artefacts of one technology are often expensive to represent by another technology. The on-line transcoding computation time can introduce additional latency that consumes a significant fraction of the speed up that the new generation of codecs could otherwise offer. Additional latency is unfortunate since the user experienced latency (without compromising on the image quality) is the main driver for a new generation of lossy image compression. The transition costs are not limited to image quality and interoperability, but organizations deploying the new coding system may need to load test and measure backend and end-user-experienced latencies, perform security analysis, redo capacity planning, possibly replace old equipment, and study the impact of the technology transition on user behavior.

This paper is structured as follows. Section 2 explains the experiments that lead the authors to focus on high quality compression. Section 3 summarizes the features that JPEG XL supports, and the motivations behind them. Finally, Section 4 explains the general architecture of the codec, and gives details and motivations behind some of its components.

In this paper we show that we have solved the high quality efficiency issues that previous formats, and particularly video formats having a second life as photography formats, tend to have. We show that we have taken several measures to reduce the migration and interoperability concerns, ranging from supporting JPEG's $8 \times 8$ DCT to enabling lossless recompression of existing JPEG images — up to a point where a file that is byte-wise identical to the original JPEG can be reconstructed from the new JPEG XL stream.

\section{MOTIVATION}

Historically, technological advances have not always been associated with a direct improvement in user experience. For example, with the initial deployment of terrestrial digital television, broadcasting companies often chose a distribution model that slightly degraded the perceived quality of the program in favor of being able to distribute more channels. Block artefacts started to appear in fast-moving complex scenes where the previously uncompressed signal had uncompromised good quality. The economic pressure of having more segmentation in the market led to a slight reduction in quality.

We would like to prevent this from happening again. Our aim is to build solutions that allow us to deliver images faster, more economically, robustly, without surprises and with a generally better perceived quality.

There was a time in the history of the internet when downgrading the quality of media was the only option for internet transfers to happen at bearable latencies. In those times, recording devices also used to produce images of much lower quality than they do today. However, we no longer live in that era. The average internet connection speed around the world is steadily increasing; it was reported at 11.03Mbps in May 2019, a 20.65\% increase compared to one year before. ${ }^{5}$ Alongside, the recording and display capabilities of current phones, cameras and monitors often have higher resolutions than an average end user can perceive. The current digital world is evolving towards richer and highly immersive user experiences, where images are expected to look natural. It is safe to say that optimizing for the quality of images should be regarded as a higher priority than optimizing for the transfer speed of lower-size images.

To find out the quality of images currently used on the web, we examined the distribution of bits per pixel (BPP) of small, medium and large JPEG images loaded by users in Google Chrome (Figure 1). The data was recorded by telemetry; it is pseudonymous and aggregated across users. Telemetry in Chrome is opt-out by default and can be disabled from the browser's "Settings" menu. We approximated the number of bytes transferred at each BPP value by multiplying the image count for a particular BPP value with the BPP value 
itself. The number of transferred bytes is important because it affects the latency and hence the user experience. Using this estimate, we calculated that between June 1st and 28th, 2019, the average BPP (weighted by the number of bytes transmitted with that BPP) was 2.97, 2.24 and 2.03 for small, medium and large images, respectively. We confirmed that there was no considerable difference between mobile and desktop platforms. This data indicates that image compression algorithms should target rich images with a relatively high BPP values, aiming to produce $1 \mathrm{BPP}$ images almost free of artefacts instead of $0.5 \mathrm{BPP}$ images with artefacts that are not too intrusive.

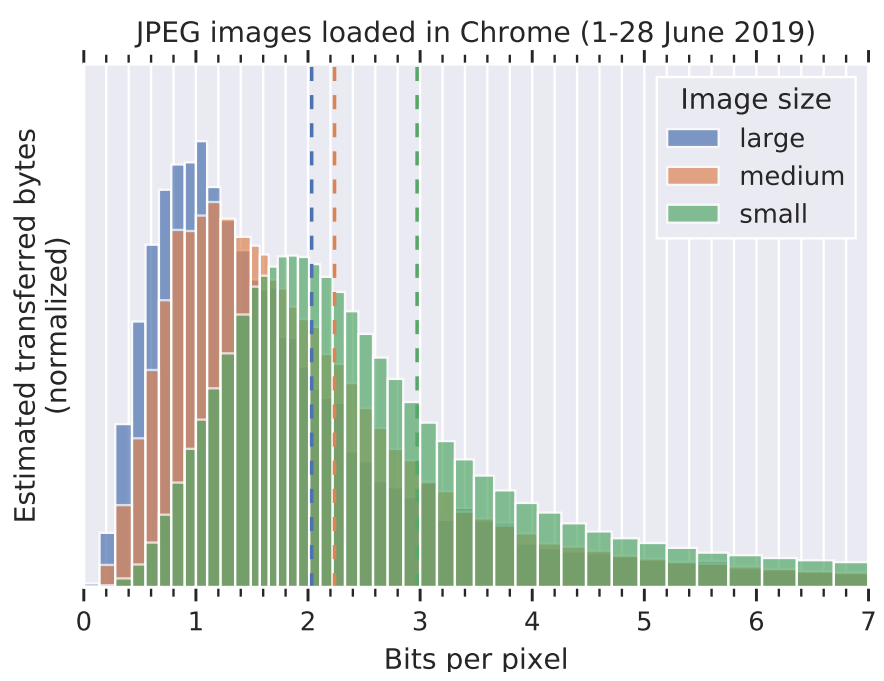

Figure 1. Estimation of bytes transferred at each BPP value. BPP values are aggregated from pseudonymous data collected by telemetry in Google Chrome. The images are classified by their smallest dimension (width or height) into small (100-400 pixels), medium (400-1000 pixels) and large (1000+ pixels). The BPP distributions are aggregated across all images loaded in Chrome across all platform from June 1st to 28th, 2019. The estimated counts are normalized for each image size category. The mean for each image size is shown with dashed vertical lines. The BPP counts are obtained from an order of billions of samples.

With these considerations in mind, JPEG XL is designed to handle high-quality images particularly well. Its aim is serving the current and future needs of an increasingly rich media experience. Ideally, an image should be compressed up to a point where there is no perceptible loss of quality from the original.

To assess the efficiency of JPEG XL over that of legacy JPEG in the task of encoding images with no perceptible loss of quality, we performed the following experiment. We selected a corpus of 43 images consisting of 12 images from imagecompression.info ${ }^{6}$ (IC dataset) and 31 images from a corpus provided by Jyrki Alakuijala $^{7}$ (J31 dataset). We chose the IC dataset because it is a standard benchmark for image compression, and the J31 dataset because it is designed to capture semantically important low intensity features on a variety of background colors, such as the fine structure of flower petals; our experience with image compression showed that red, purple, and magenta backgrounds often cause high frequency features to be lost and that image quality metrics have difficulty normalizing the differences across different background colors. We used all images from each dataset, except for leaves_iso_1600 and nightshot_iso_100 from IC, which had duplicates at a different ISO level. As the IC images are very large, we rescaled them so that the maximum dimension would be 1024 pixels. All IC images and 6 J31 hence corresponded to the large size category described above, while the other 25 J31 images corresponded to the medium size category.

We compressed each of the 43 images with legacy JPEG and JPEG XL at 24 different quality levels. For images compressed with JPEG, we used libjpeg1.5.2 with a quality parameter of 40,44, 48, 52, 56, 60, 64, $68,72,76,80,82,84,86,88,90,92,94,95,96,97,98,99$ and 100 for each of the 24 images respectively. For images compressed with JPEG XL, we used default settings on the latest development version of the codec, and 
a distance value* of $8.0,6.0,4.0,3.0,2.8,2.6,2.4,2.2,2.0,1.8,1.6,1.5,1.4,1.3,1.2,1.1,1.0,0.9,0.8,0.7,0.6$, $0.5,0.4$ and 0.3 for each of the 24 images respectively. For each image and compression method, we recorded the corresponding number of bytes $b$ and computed the bits per pixel (BPP) for that image (as $8 b /(x \cdot y)$ for an image of width $x$ and height $y$ pixels). Finally, we decompressed the images to PNG to allow the browser to display them without further loss.

We asked 36 healthy adult participants to indicate the lowest quality that they would find acceptable to compress pictures with no perceptible loss of quality. All participants had normal or corrected vision and were instructed to adjust their monitor position and chair height in order to look at the images in a comfortable and natural position. The images were displayed on a 27" QHD monitor with a resolution of $2560 \times 1440$ on the default brightness setting. All assessments were performed during daylight in a room lit by indirect sunlight. The order of the four sets and conditions (IC JPEG, IC JXL, J31 JPEG, J31 JXL) was randomised, but always so that the two IC sets and the two J31 sets were presented in consecutive order. For each image, the assessment was performed by moving a slider with 25 positions to the left or to the right to adjust the compression level of the image. The rightmost position was the starting position and it displayed the uncompressed image. The leftmost position corresponded to the lowest compression quality and the following 23 positions corresponded to gradually increasing compression quality levels specified above.

Out of the 3096 datapoints collected in total, we discarded 13 datapoints that were invalid due to a problem in recording the responses that we only found after performing the experiment. As this involved a very small number of points, we treated these as missing and discarded them.

We found that a lower BPP value was consistently selected for JPEG XL compared to legacy JPEG (Figure 2). Pooling the results from both datasets together, the mean selected BPP was 2.09 for legacy JPEG, which is in agreement with the Chrome statistics above. The JPEG XL distribution mode (computed using bins of size 0.1), mean, median and skewness were $48 \%, 46.27 \%, 41.33 \%$, and $54.7 \%$ lower than those of legacy JPEG. This effect was preserved when splitting across sets (Figure 3) and participants (Figure 4).

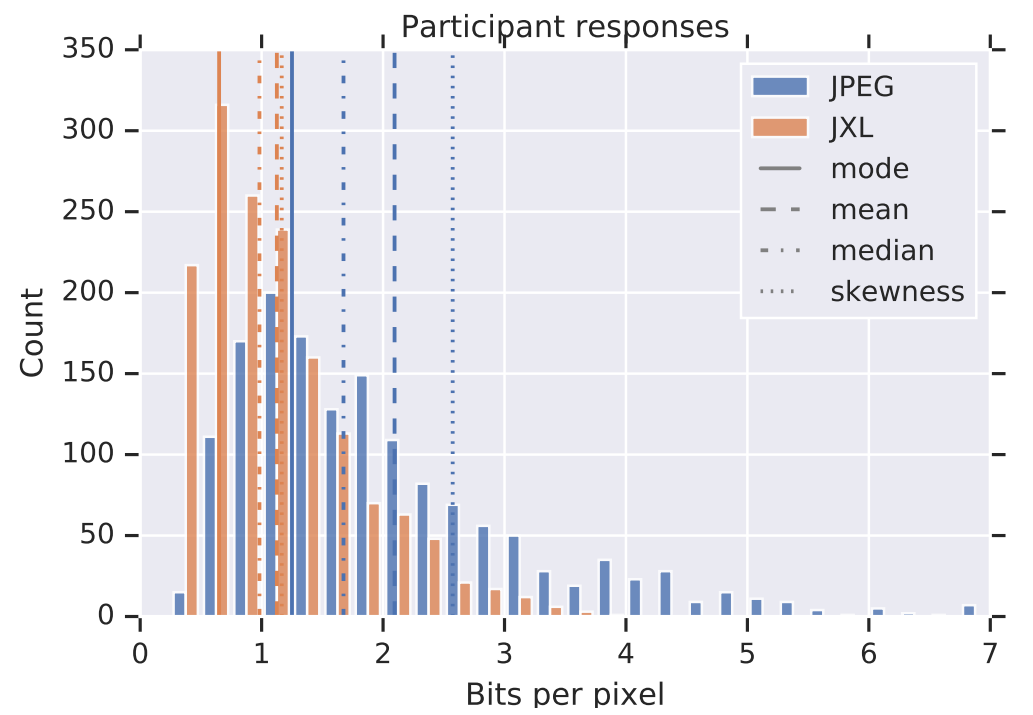

Figure 2. All participant responses groups by condition (JPEG and JPEG XL). The mode was obtained by grouping the data into bins of size 0.1 . The histogram bin size is 0.25 . For JPEG, an additional 28 responses larger than 7 , with a maximum of 13.08 , are not shown here.

*approximately proportional to the inverse of the quality parameter of legacy JPEG, calibrated so that a distance of 1.0 corresponds to barely perceptible artefacts under normal viewing conditions 


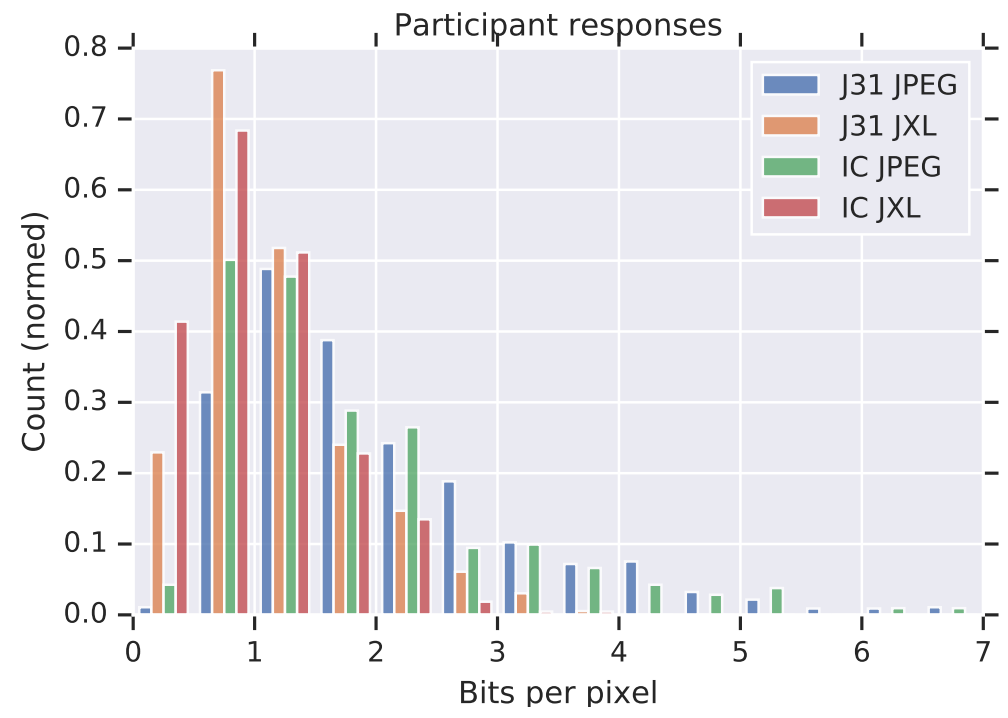

Figure 3. All participant responses grouped by condition (JPEG and JPEG XL). The histogram bin size is 0.5 . The values are normalized so that their sum per condition is $1.0 /$ bin_size $=2.0$. For JPEG, the additional responses larger than 7 and not shown here sum to 0.07 .

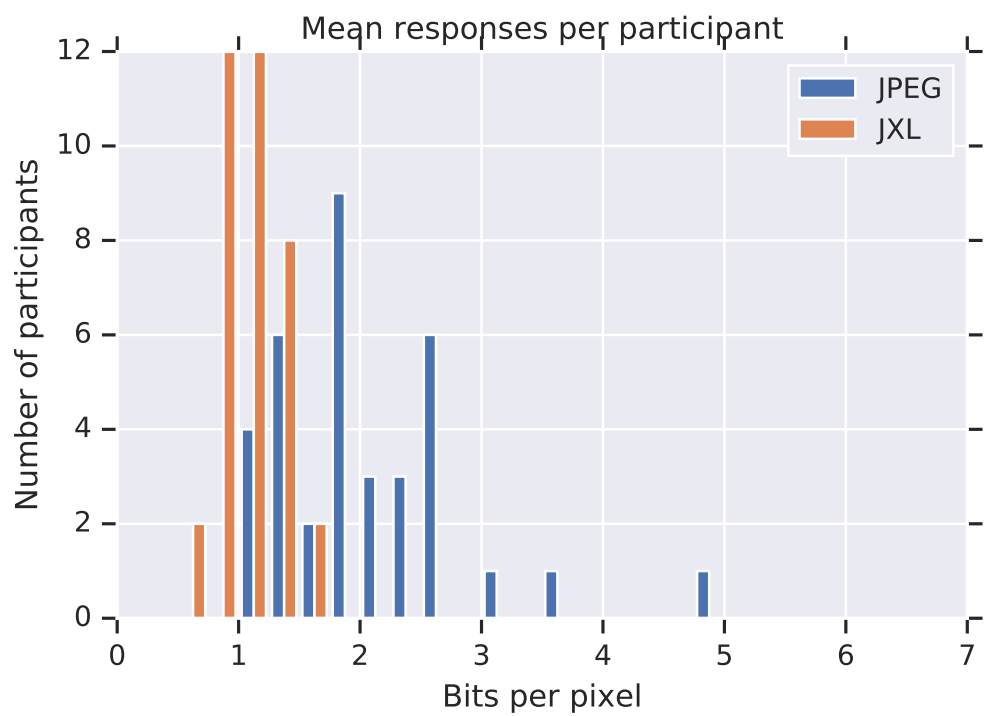

Figure 4. Mean responses by participant and condition (JPEG and JPEG XL). For each participant, each value was obtained by averaging over individual dataset (IC and J31), then averaging the two values. The histogram bin size is 0.25 .

These results indicate that JPEG XL yields a large improvement in the representation efficiency of highquality images, where no perceptible loss of quality is desired. By replacing legacy JPEG with JPEG XL, it is possible to represent such images while almost halving the required number of bits.

\section{CODEC FEATURES}

JPEG XL aims to be a full-featured image codec, with support for all the features that are expected by modern image formats. Thus, JPEG XL supports an arbitrary number of channels, including alpha and depth channels; it also allows parallel, progressive, and partial decoding. Moreover, JPEG XL supports encoding of multiple animation frames, with different behaviours for how each frame should be blended with the previous ones: they can be summed, replace the full frame or a sub-rectangle, or alpha-blended. JPEG XL also supports a fully lossless mode and a responsive-by-design mode. 
Websites are viewed on a large variety of devices. The concept of responsive images addresses the different needs of phones, tablets, computers, smart watches etc. that was traditionally addressed by having separate website versions for different devices. The responsive image feature is however an additional requirement and it limits the format designers' ability to make other compromises related to image quality and decoding speed. In JPEG XL we have overcome these limitations by having a dedicated mode, modular mode, for the most responsive use. The modular mode uses a Haar-like integral transform that can exactly recover all subresolution images, various color space transforms to be able to better represent existing images and achieve otherwise better compression ratios through decorrelation, and an adaptive context model to improve the efficiency of the entropy coding step.

The two common ways to model color vision are trichromatic theory and the opponent-process theory. The LMS colorspace represents the response of three types of cones in the human retina that are named for their sensitivity at long (L), medium (M), and short (S) wavelengths. In JPEG XL we base color modeling on a possibly new hybrid color theory, where we use the opponent-process theory for L and M receptors, and the trichromatic theory for $\mathrm{S}$ receptors. This allows us to control quantization and decorrelation of $\mathrm{S}$ receptors separately from the $\mathrm{L}$ and $\mathrm{M}$ receptors. There is lesser spatial density of the $\mathrm{S}$ receptors than of the $\mathrm{L}$ and $\mathrm{M}$ receptors, leading to a lesser information density of respective perceptual fields, and the separation allows us to accomodate for that in how we store colors. We call this colorspace XYB, where $\mathrm{Y}$ is the sum of L and $\mathrm{M}$ signaling after reception and $\mathrm{X}$ is the difference. $\mathrm{B}$ represents the signaling of the $\mathrm{S}$ receptors. In JPEG XL we model the compression through storing the cubic root of the value over the receptor's spectral efficiency. While this does not exactly match receptors' compression properties — which are modeled as hybrid-log-gamma in some other modern efforts - it is practical since it is fast to decode and the error of the receptor response and cubic root can be stored in the adaptive quantization field, giving roughly equally efficient quantization performance at a higher decoding speed.

\section{CODEC ARCHITECTURE}

This section describes the architecture of the JPEG XL image codec, as per the committee draft of the specification. ${ }^{9}$ An overview is given in Figure 5; the rest of the section gives more details about each component.

\subsection{Integral transforms}

JPEG $^{1}$ is fundamentally based on the $8 \times 8$ DCT-II algorithm by Arai, Agui and Nakajima. ${ }^{10}$ Variable-DCT mode of JPEG XL is still based on fundamental $8 \times 8$ block units, but extends this approach, and allows the use of any bidimensional DCT-II transform whose sides are one of 8,16 , or $32^{\dagger}$. Doing so allows further reductions in the entropy of the image for homogeneous areas; on the other hand, it creates artefacts with longer reach, and thus the use of these transforms requires careful selection on the encoder side.

JPEG XL also supports the use of "special" transforms that cover specific use cases:

- small DCTs of sizes $8 \times 4,4 \times 8,4 \times 4$, and $2 \times 2$ cover the opposite use case of larger transforms, such as very heterogeneous areas, for which it is important to reduce the propagation distance of artefacts. Note that these small DCTs are still applied on $8 \times 8$ blocks: as an example, for the $4 \times 4$ DCT, the transform is applied on each of the four $4 \times 4$ areas in the $8 \times 8$ block; coefficients are then mixed in such a way that DC ends up in the top-left $2 \times 2$ corner of the block, and a $2 \times 2$ DCT is applied on the DC coefficients to obtain a single DC value. Similar techniques are used to obtain a DC value for other DCTs.

- the IDENTITY transform covers the case of some pixels in the block having very different values from nearby pixels. As for the $4 \times 4 \mathrm{DCT}$, it applies a transform to each of the four $4 \times 4$ areas in the $8 \times 8$ block, and then proceeds with shuffling the resulting coefficients and doing a $2 \times 2 \mathrm{DCT}$ on the DC coefficients.

\footnotetext{
${ }^{\dagger}$ DCT sizes other than 8 use a different DCT algorithm, described by Vashkevich and Petrovsky ${ }^{11}$
} 


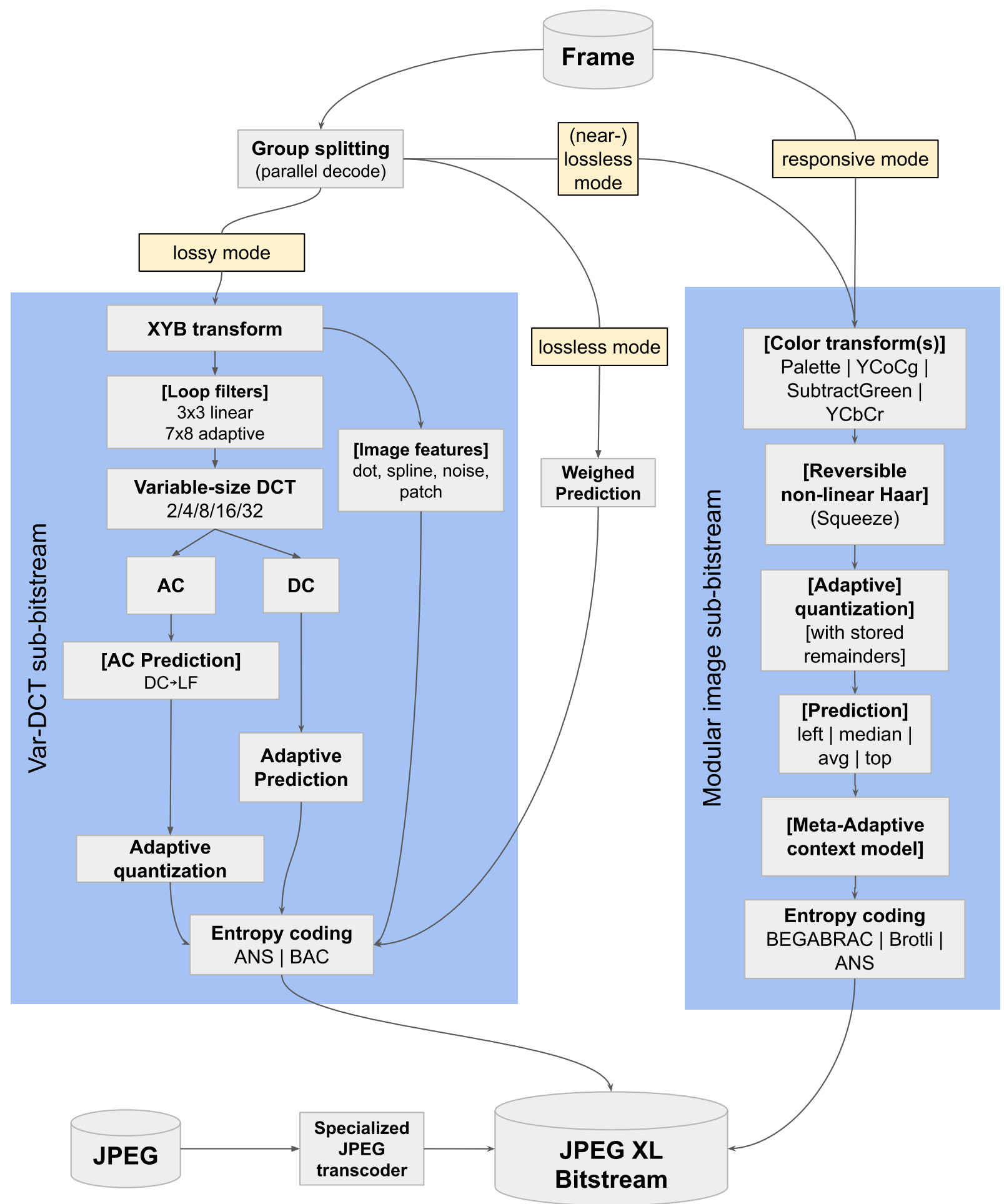

Figure 5. Block diagram architecture of JPEG XL. 
However, the $4 \times 4$ transform that is used is not a DCT. Instead, the transform computes the average of the $4 \times 4$ block and, for all other values of the block except the one in position $(1,1)$, the difference between the value and the one in position $(1,1)$. Storing deltas compared to one pixel instead of deltas from the average avoids accumulating quantization error on the missing pixel, while choosing pixel $(1,1)$ instead of $(0,0)$ provides slightly better compression because it is more correlated with the values in the $4 \times 4$ block, on average.

- the AFV transform is also similar to $4 \times 4$ DCT and IDENTITY, but with a different transform for each $4 \times 4$ area. In particular, AFV computes a basis change similar to what a DCT does, but with specialized basis functions that allow the 3 corner pixels to be stored separately. This allows to easily encode blocks that have an edge barely cutting through them, which gives a very different value to a few pixels in one of the corners.

As with JPEG, JPEG XL also has special handling of DC coefficients. For transforms that are bigger than $8 \times 8$, this creates a problem: there is now only one DC coefficient that spans more than one $8 \times 8$ block. JPEG XL handles this situation by computing $\frac{X}{8} \times \frac{Y}{8}$ pseudo- $D C$ coefficients for each $X \times Y$ transform, roughly corresponding to the average value in each $8 \times 8$ block, in a reversible way. This can be done thanks to the following key observation:

Lemma 4.1. Given $N$ and $K \leq N$, there exist non-zero constants $c_{0}, \ldots, c_{2^{N-K}-1}$ such that the following two procedures are equivalent for any sequence of numbers $a_{0}, \ldots, a_{2^{N-K}-1}$ :

1. Compute an inverse DCT of size $2^{N-K}$ on the array $c_{0} a_{0}, \ldots, c_{2^{N-K}-1} a_{2^{N-K}-1}$

2. Append $2^{N-K} \cdot\left(2^{K}-1\right)$ elements with a value of 0 to the $a_{0}, \ldots, a_{2^{N-K}-1}$ array, compute an inverse DCT of size $2^{N}$ on the resulting array, and replace each of the $2^{N-K}$ consecutive groups of $2^{K}$ values with their average.

For example, with $N=4$ and $K=3$, both procedures compute the same two values, the average of each of the two halves of a IDCT of size 16 computed on an array with 2 nonzero entries followed by 14 zero entries.

This claim can easily proven by induction on $K$. By choosing $K=3$, and applying the lemma along both dimensions, this allows us to compute a value that is approximately the average of each $8 \times 8$ block covered by a large DCT (computed on pixel values after ignoring some higher frequencies) that also allows to completely recover an equivalent number of low-frequency DCT coefficients, as the first of the two equivalent procedures in Lemma 4.1 is clearly reversible.

\subsection{Entropy coding}

The legacy JPEG format offers the choice of using Huffman coding ${ }^{12}$ or Arithmetic Coding ${ }^{13}$ as an entropy coder. Both of these options come with disadvantages: Huffman coding is sub-optimal when symbol probabilities are not exact powers of two, and Arithmetic Coding implementations typically have fairly slow decoding speed.

JPEG XL uses Asymmetric Numeral Systems ${ }^{14}$ (also known as ANS), a recently-introduced entropy coder that achieves compression ratios similar to Arithmetic Coding, while being significantly faster during decoding.

JPEG XL encodes in a single ANS stream symbols drawn from multiple different probability distributions. Two techniques are used to give more flexibility to encoders: distribution clustering and variable-precision distribution encoding.

A single ANS stream may contain symbols from thousands of different distributions. Encoding all of these distributions would use a significant amount of bits, making this choice sub-optimal for all but the longest of streams. Thus, JPEG XL allows defining clusters of similar distributions, with a single histogram being encoded for the whole cluster. This allows more flexibility than simply using fewer distributions, as the clustering can be defined per-file instead of being fixed. The encoding of the clusters uses a move-to-front transform and run-length encoding to minimize the bitstream overhead. 
To further reduce the overhead for relatively small clusters, the encoder can choose between multiple different representations for the distributions themselves: a simple "flat" representation (that produces histograms with roughly uniform probabilities), useful for short streams, and a representation that stores, for each probability value, its magnitude and a variable number of less-significant bits, controllable on a per-histogram basis, that allows to further control the tradeoff between histogram and stream size.

Finally, to reduce the number of distinct symbols that get encoded in an ANS stream, which has beneficial effects on both histogram size and decoding speed of the entropy coder, many parts of JPEG XL use a hybrid unsigned integer encoding scheme: values below 16 are encoded as symbols directly, while any other value is encoded with a symbol that allows to recover the highest 2 bits set; other bits are stored uncompressed.

\subsection{Color correlation}

From the definition of the XYB colorspace, it follows that a fully gray pixel will be represented as a multiple of $(0,1,0.935669)$. Keeping this representation as-is is undesirable, as it transmits the luma information on two channels at the same time. Thus, JPEG XL applies a linear transformation to pixel values immediately after dequantization, adding a multiple of the $\mathrm{Y}$ channel to the $\mathrm{X}$ and $\mathrm{B}$ channels.

In particular, the default correlation factor is 0 for the $X$ channel and 0.935669 for the $B$ channel: this allows to transmit luma information using the $Y$ channel only.

However, these factors are not necessarily optimal on areas of the image with a strong chroma component (such as, for example, fully red regions). Therefore, JPEG XL allows altering the correlation factors locally, for tiles of $64 \times 64$ pixels, with a dedicated control field.

\subsection{Adaptive quantization}

JPEG only allows the choice of a single quantization matrix (per channel) for the whole image. This leads to the same amount of quantization everywhere, even if some areas have more detail and may thus benefit from increased quantization.

In JPEG XL, the choice of quantization matrix is still global (for each given integral transform); however, this quantization matrix can be scaled locally, to decrease artefacts in more "complex" areas without increasing the amount of bits used in other parts of the image.

Combined with a measure of loss, this allows encoders to target a roughly uniform amount of loss across the image, avoiding large variations.

\subsection{Adaptive predictor}

To improve compression ratio, legacy JPEG subtracts the value of the DC coefficient for the previously encoded block from the DC coefficient of the current block. Because of local similarities displayed by typical images, this results in significant savings.

However, this prediction mode is fairly primitive, and does not take into account the bidimensional nature of images. To address this concern, JPEG XL uses a bidimensional "adaptive" predictor, that chooses between eight different prediction modes, based on their behaviour on nearby pixels. This predictor is used for DC encoding, but also for the encoding of various control fields.

More specifically, when predicting a value in position $(x, y)$, the adaptive predictor looks at the maximum error that each prediction mode would have produced on pixels $(x-1, y),(x, y-1)$ and $(x-1, y-1)$, and chooses the prediction mode that is expected to produce the least amount of error. If the expected error computed this way is 0 , the adaptive predictor also keeps track of the number of prediction modes that are expected to reach this accuracy level. The expected error and the number of "correct" prediction modes is used to split the encoded residuals into multiple distributions. This allows significant size savings, since this procedure detects flat areas in the image and uses specific distributions for them.

A variation of this predictor is used for lossless mode. The lossless predictor uses fewer prediction modes (four instead of eight), but computes a weighted average of the predictions instead of choosing one of them. 

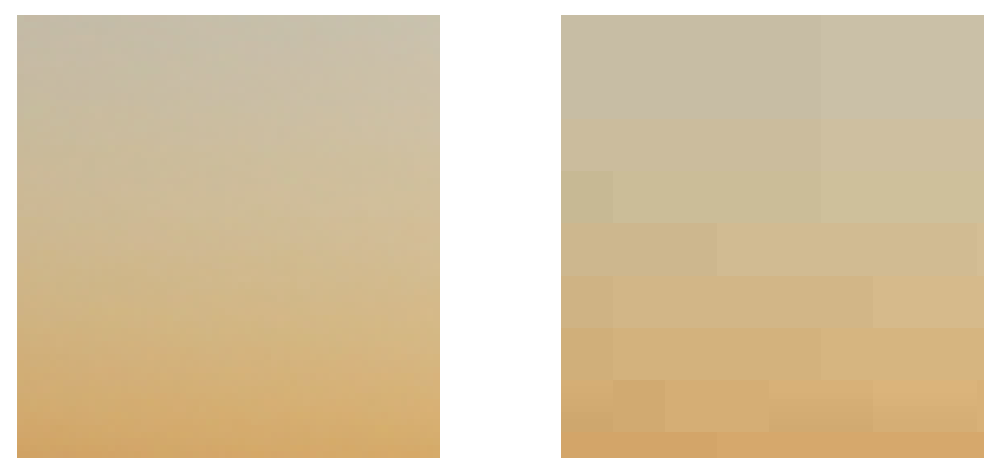

Figure 6. Example of banding on low quality images: left original, right compressed image.

\subsection{DC handling}

One of the most noticeable artefacts produced by JPEG compression at lower quality is banding, the transformation of an area of the image containing a slowly varying color (such as the sky at sunset) into one that contains a few bands of the same color (see Figure 6). This creates very noticeable artefacts at the boundaries between two different bands.

Banding in JPEG is typically caused by DC quantization. To avoid this effect, JPEG XL allows using finer quantization steps for encoding residuals that are close to 0 in the original image. This is in practice equivalent to using finer quantization steps in areas that are slowly varying, but without sacrificing compression ratio as much as using finer quantization on the whole image would do.

To further reduce banding in areas with steeper gradients, JPEG XL applies a selective smoothing algorithm to the DC image, that is only allowed to move values inside their quantization boundaries. If the smoothed value would be outside of the quantization boundaries, it is discarded, and the original is used.

\subsection{LF predictions}

To reduce banding further, and to make block artefacts less noticeable in the resulting image, JPEG XL estimates the low-frequency coefficients of each $X \times Y$ transform (the top-left corner of size $\frac{X}{4} \times \frac{Y}{4}$, excluding the $\frac{X}{8} \times \frac{Y}{8}$ corner) from the DC image. This procedure starts by extending the known $\frac{X}{8} \times \frac{Y}{8}$ coefficients to have size $\frac{X}{4} \times \frac{Y}{4}$, by filling missing coefficients with zeros. It then uses the inverse of the first procedure described in Lemma 4.1 on each block to produce a $2 \times$ upsampled version of the DC image.

After applying the a smoothing algorithm similar to the one used on the DC image, the upsampled DC is converted back to DCT coefficients; the low-frequency values that are produced this way are then added to the encoded low-frequency coefficients.

\subsection{AC encoding}

JPEG always encodes AC coefficients using the so-called zig-zag order, which proceeds in order of increasing sum of coordinates in the block.

As JPEG XL uses different transform sizes, it generalizes the zig-zag order to those cases. Moreover, since this order is not necessarily optimal for a given image, it allows to encode a custom order. This custom order is encoded as a permutation of the zig-zag order, using a Lehmer ${ }^{15}$-like code to achieve efficient encoding of the identity permutation.

The encoding of AC coefficients proceeds in the order specified above; it keeps track of the number of remaining non-zeros in the current block, using that value, combined with the current position, to split coefficients into multiple distributions. No further values are present in the bitstream when it is known that no non-zero coefficient is left. 

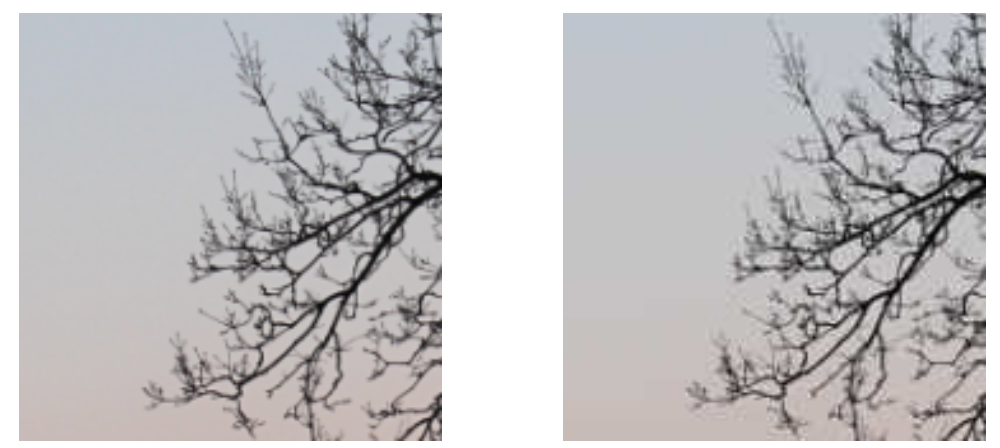

Figure 7. Example of ringing on low quality images: left original, right compressed image. Because of ringing, the sky is not flat in the regions close to the tree branches.

\subsection{Loop filters}

Despite the significant improvements that JPEG XL delivers to reduce artefacts, block boundaries and ringing can still be noticed, especially at somewhat lower qualities. Ringing artefacts are spurious signals near sharp edges, caused by quantizing or truncating high-frequency components (see Figure 7). To mitigate their impact, JPEG XL employs two different loop filters that are applied to the image after the decompression process.

The first loop filter is a smoothing convolution. As smoothing the image inherently introduces a sharpness loss, this effect is compensated by the encoder by means of a sharpening filter that is applied before the DCT step. The overall effect of this procedure is that the visual impact of block boundaries gets reduced, while still preserving sharp details present in the original image.

The second loop filter is intended to reduce ringing, while still preserving texture that is transmitted in the image. To achieve this effect, it applies an adaptive smoothing algorithm related to Non-Local Means ${ }^{16}$ with some modifications to improve processing speed. To further improve the detail preservation of this filter, the JPEG XL format can require the decoder to apply a quantization constraint to the output of the filter: the DCT step is applied again, and the decoder ensures that the resulting coefficients are inside the range of values that would have been quantized to the coefficient read from the bitstream by clamping. The decoder then applies an IDCT step again to produce the final output of the filter.

\subsection{Image features}

Certain image features can pose challenges to the DCT and are sometimes best handled by dedicated tools. This section presents a few such encoding tools that are specified by JPEG XL.

One such technique that JPEG XL allows is to draw splines on top of the decoded image by means of addition in XYB space. More specifically, they consist in an approximation of centripetal Catmull-Rom splines, along which evenly-spaced (1 pixel apart) strokes of a Gaussian brush are applied. The color of the brush and the variance of the Gaussian kernel can vary along the spline and are each encoded using the DCT32 transform.

Another technique concerns dots, by which we mean one or very few pixels that are visibly very different from their neighboring pixels. At lower quality, due to the nature of DCT transforms used by JPEG XL, the disappearance of dots might be a noticeable artefact. To mitigate this effect, JPEG XL allows to draw dots that get summed to the decoded images. Those dots are modeled as bidimensional Gaussians, which may have an elliptical shape, with various rotations of the axes and intensities.

JPEG XL allows to add patches to an image. Patches are small pixel patterns. When those patches appear multiple times in an image, it might be beneficial to store them only once, together with the positions where they appear. This can be used for example to improve the appearance of compressed printed text in an image, which is also problematic to represent in a DCT-based codec.

There is tendency that noise, when present in the original image, is lost when compressed. To recover more realistic looking images, JPEG XL allows to add noise to the image. We employ an intensity dependent noise model. ${ }^{17}$ A pseudorandom procedure generates the noise that is added to the image. 


\subsection{Group splitting}

If the image to be encoded is large enough (more than 256 pixels along any axis), it is split into sub-rectangles of size at most $256 \times 256$, which are encoded independently. The bitstream also contains an index of the bitstream positions of those rectangles that allows decoders to seek to the start of each.

This gives four benefits: it allows decoders to process each rectangle independently in parallel, to decode only selected regions of large images, to rearrange rectangles (e.g. in saliency order) and to restart decoding if a prior rectangle is corrupted.

\subsection{DCT-based progressive image}

The JPEG XL bitstream can contain AC coefficients in multiple passes that are summed together. This allows to progressively encode images, by sending low-frequency coefficients first, and higher frequency coefficients later. It is also possible to refine quality in selected regions of the image in a given pass, allowing area-of-interest progressive decoding.

DC coefficients are always sent first, so it is always possible to obtain an $8 \times$ downsampled image from the JPEG XL bitstream without reading the full file.

\subsection{JPEG recompression}

JPEG recompression allows smooth migration from legacy JPEG to JPEG XL: existing JPEG files can be losslessly transformed to the newer and more compact format.

The legacy JPEG format has been thoroughly explored ${ }^{18,19}$ over the years and most of its inefficiencies are addressed in the JPEG XL recompression format:

- more robust DC coefficient prediction is used

- $\mathrm{AC} 0 \mathrm{x}$ and $\mathrm{ACx} 0$ coefficients are predicted on the base of neighboring blocks

- Huffman entropy encoding is replaced with ANS and Binary Arithmetic coding

- frequently used ICC profiles, Huffman code tables, and quantization tables are encoded as template parameters

- context modeling is used to separate entropy sources

- similar to the approach described in Section 4.8, DCT coefficients are reordered in a such way that more blocks have longer series of zeros at the end. The index of last non-zero coefficient is encoded explicitly, which is more efficient than limited RLE.

Those improvements enable $16 \%$ size savings on average in a corpus of 100000 random images from the Internet. For larger photographic images, the savings are in the range $13 \%-22 \%$, depending on the JPEG encoder and quality settings.

\section{CONCLUSIONS}

This paper introduces the coding tools used in JPEG XL: a new approach for responsive images, and a variablesize DCT designed from the ground up for economical storage of high-quality images.

Large-scale user metrics indicate that most use cases on the internet target higher quality levels than previously assumed in codec design. This helps explain why codecs optimized for lower bitrates have not supplanted the 27-year-old JPEG standard. We present new quality-of-experience data indicating JPEG XL requires less than half the bitrate of JPEG to achieve perceptually lossless storage, with fewer outliers. In other experiments, we have found JPEG XL encodings to be $33-40 \%$ of the size of libjpeg output at similar psychovisual quality.

We acknowledge that JPEG and JPEG XL will co-exist. Rather than reduce the quality of existing images by transcoding, or increase hosting costs by storing both formats, we propose to build a bridge between JPEG 
and JPEG XL. The coding tools are carefully designed to interoperate with JPEG, allowing us to shrink existing images without quality loss and create new images that can be transcoded back to JPEG. This creates a gradual update path which benefits both hosts and users at each step.

We believe this fresh approach will overcome previous difficulties in replacing the highly successful JPEG, GIF, and PNG formats, and help establish a modern format that simplifies image serving, improves quality and reduces user-experienced latency.

\section{REFERENCES}

[1] Wallace, G. K., "The JPEG still picture compression standard," IEEE transactions on consumer electronics 38(1), xviii-xxxiv (1992).

[2] "Final call for proposals for a next-generation image coding standard (JPEG XL)." https://jpeg.org/ downloads/jpegxl/jpegxl-cfp.pdf. Accessed 2019-07-26.

[3] "Github - google/pik: A new lossy/lossless image format for photos and the internet." https://github. com/google/pik. Accessed 2019-07-26.

[4] "Github - cloudinary/fuif: Free universal image format." https://github.com/cloudinary/fuif. Accessed 2019-07-26.

[5] "Worldwide broadband speed league 2019." https://www.cable.co.uk/broadband/speed/ worldwide-speed-league/. Accessed: 2019-07-25.

[6] "Image compression benchmark." http://imagecompression.info/. Accessed: 2019-07-25.

[7] Alakuijala, J., "Image compression benchmark." https://drive.google.com/corp/drive/folders/OBOw_ eoSgaBLXY1J1YUVOMzM5VFk. Accessed: 2019-07-25.

[8] "libjpeg 1.5.2 release." https://github.com/libjpeg-turbo/libjpeg-turbo/releases/tag/1.5.2. Released 2017-08-09.

[9] Rhatushnyak, A., Wassenberg, J., Sneyers, J., Alakuijala, J., Vandevenne, L., Versari, L., Obryk, R., Szabadka, Z., Kliuchnikov, E., Comsa, I.-M., Potempa, K., Bruse, M., Firsching, M., Khasanova, R., van Asseldonk, R., Boukortt, S., Gomez, S., and Fischbacher, T., "Committee draft of JPEG XL image coding system," (2019).

[10] Arai, Y., Agui, T., and Nakajima, M., "A fast DCT-SQ scheme for images," IEICE TRANSACTIONS (1976-1990) 71(11), 1095-1097 (1988).

[11] Vashkevich, M. and Petrovsky, A., "A low multiplicative complexity fast recursive DCT-2 algorithm," arXiv preprint arXiv:1203.3442 (2012).

[12] Huffman, D. A., "A method for the construction of minimum-redundancy codes," Proceedings of the IRE 40(9), 1098-1101 (1952).

[13] Rissanen, J. and Langdon, G. G., "Arithmetic coding," IBM Journal of research and development 23(2), 149-162 (1979).

[14] Duda, J., Tahboub, K., Gadgil, N. J., and Delp, E. J., "The use of asymmetric numeral systems as an accurate replacement for Huffman coding," in [2015 Picture Coding Symposium (PCS)], 65-69, IEEE (2015).

[15] Laisant, C.-A., "Sur la numération factorielle, application aux permutations," Bulletin de la Société Mathématique de France 16, 176-183 (1888).

[16] Buades, A., Coll, B., and Morel, J.-M., "A non-local algorithm for image denoising," in [2005 IEEE Computer Society Conference on Computer Vision and Pattern Recognition (CVPR'05)], 2, 60-65, IEEE (2005).

[17] Khasanova, R., Wassenberg, J., and Alakuijala, J., "Noise generation for compression algorithms," CoRR abs/1803.09165 (2018).

[18] Lakhani, G., "DCT coefficient prediction for JPEG image coding," in [2007 IEEE International Conference on Image Processing], 4, IV - 189-IV - 192 (Sep. 2007).

[19] Richter, T., "JPEG on STEROIDS: Common optimization techniques for JPEG image compression," in [2016 IEEE International Conference on Image Processing (ICIP)], 61-65 (Sep. 2016). 